\title{
Desarrollo de aplicación web para automatizar la gestión de procesos del programa educativo de ISC de la UPFIM
}

\section{Development of a web application to automate the process management of the UPFIM ISC educational program}

REYNA-ANGELES, Omar†*', HERNÁNDEZ-TAPIA, Zaila, HERNÁNDEZ-LEÓN, Jonathan Misael y GARCÍA-ROMO, Everardo Said

Universidad Politécnica de Francisco I. Madero, México.

ID $1^{\text {er }}$ Autor: Omar, Reyna-Angeles / ORC ID: 0000-0001-6604-9059, Researcher ID Thomson: I-3308-2018, CVU CONACYT ID: 097627

ID $1^{\text {er }}$ Coautor: Zaila, Hernández-Tapia / ORC ID: 0000-0003-2564-3451, Researcher ID Thomson: G-6592-2018, CVU CONACYT ID: 622127

ID $2^{\text {do }}$ Coautor: Jonathan Misael, Hernández-León / ORC ID: 0000-0002-5475-9624, Researcher ID Thomson: AAP-72802020

ID $3^{\text {er }}$ Coautor: Everardo Said, García-Romo / ORC ID: 0000-0002-6086-1529, Google Scholar ID: pgfdvy0AAAAJ

DOI: $10.35429 /$ JIT.2020.24.7.19.22

Recibido: Julio 25, 2020; Aceptado Diciembre 30, 2020

\section{Resumen}

Se presenta el desarrollo de una aplicación web modular para automatizar la gestión de los procesos de recursos materiales de la dirección del programa educativo de la Ingeniería en Sistemas Computacionales (ISC) de la Universidad Politécnica de Francisco I. Madero (UPFIM) y la información generada en la vinculación con padres de familia, con el propósito de optimizar tiempos y materiales, además de contar con información que permita generar automáticamente estadísticas y reportes para análisis pertinentes y toma de decisiones, considerando a futuro la integración de otros módulos. La metodología que se utilizará es una propia definida con anterioridad basada en principios de metodologías ágiles ya que se cuenta con elementos claves para implementarla. Este trabajo se pretende implementarlo de manera interna en la dirección del programa educativo de ISC para después compartirlo con los demás programas de la UPFIM

Automatización de gestión de procesos, Integración de procesos, Software propio

\begin{abstract}
This presents the development of a modular web application to automate the management of the material resource processes of the principal's office of the educative program of Computer Systems Engineering (ISC Spanish Acronym) of the Universidad Politécnica de Francisco I. Madero (UPFIM) and the generated information linked with parents, in order to optimize time and materials. In addition to having information that automatically generates statistics and reports for pertinent analysis and decision-making, considering the unification of other modules in the future. The methodology to be used is its own previously defined based on principles of agile methodologies since it has key elements to implement it. This work intends to be implemented within the ISC educative program office and then shared with the other UPFIM programs.
\end{abstract}

Process management automation, Process integration, Own software

Citación: REYNA-ANGELES, Omar, HERNÁNDEZ-TAPIA, Zaila, HERNÁNDEZ-LEÓN, Jonathan Misael y GARCÍAROMO, Everardo Said. Desarrollo de aplicación web para automatizar la gestión de procesos del programa educativo de ISC de la UPFIM. Revista de Tecnologías de la Información. 2020. 7-24:19-22.

\footnotetext{
* Correspondencia del Autor (Correo electrónico: oreyna@upfim.edu.mx)

$\dagger$ Investigador contribuyendo como primer autor.
} 


\section{Introducción}

En la dirección del programa educativo de ISC de la UPFIM se llevan a cabo procesos administrativos que se consideran apoyo para la formación académica de los alumnos, actualmente la mayoría de estos se realizan de forma manual generando gasto de papel, además de la inversión de tiempo significativo en la generación de estadísticas que son utilizadas para los análisis de los procesos y sus resultados, esto afecta la toma de decisiones, limitando que sean oportunas para la implementación de acciones o estrategias que impacten en la mejora de la formación académica

Por otro lado, al tener procesos manuales se complica la integración sistemática de estos.

El gasto de papel es algo que se ha buscado disminuir sustancialmente en la UPFIM debido que actualmente está en proceso de seguimiento para recertificación de su Sistema de Gestión Ambiental (SGA) basado en la norma ISO 14000:2015

Uno de los procesos de apoyo sustanciales en la ISC de la UPFIM es la vinculación con padres de familia, ahí se integran las siguientes acciones

a) Reuniones con padres de familia.

b) Atención a padres de familia vía telefónica.

c) Atención a padres de familia por correo electrónico.

d) Atención a padres de familia de manera personal.

Este proceso tiene relación con otros que se realizan a nivel institucional como tutorías y asesorías, atención de alumnos en riesgo, entre otros; en la dirección de ISC se relaciona con atención de alumnos, atención de docentes y administración de recursos materiales del programa educativo.
La automatización de la gestión de estos procesos es necesaria, es por eso que se ha decidido que participen docentes y alumnos del programa educativo en el desarrollo de una aplicación web que integre los procesos internos para después buscar la integración con los generales de la institución.

Se pretende que sea modular y se definan módulos específicos para cada proceso, además que la aplicación sea web para que tenga buen nivel de accesibilidad.

Los módulos que se consideran para este trabajo son:

- Vinculación con padres de familia.

- Recursos materiales.

\section{Metodología}

La metodología que se siguió fue una definida con anterioridad para todo el software que se desarrolla en la ISC, está basada en principios ágiles ya que se cuenta con elementos propicios para su implementación, como son:

a) Comunicación cercana con el cliente.

b) Buena comunicación entre integrantes del equipo.

c) Conocimiento del lenguaje de programación y gestor de base de datos a utilizar por parte de los desarrolladores.

d) El software a desarrollar se puede seccionar en módulos.

e) Documentación mínima requerida debido al conocimiento de los procesos por parte de los desarrolladores.

En la tabla 1 se presentan las etapas de la metodología y los artefactos generados para cada una de ellas. 


\begin{tabular}{|l|l|}
\hline \multicolumn{1}{|c|}{ Etapa } \\
\hline \multirow{4}{*}{$\begin{array}{l}\text { Concepción } \\
\text { General }\end{array}$} & $\begin{array}{l}\text { Esquema general de la aplicación por } \\
\text { procesos }\end{array}$ \\
\cline { 2 - 2 } & Plan general de desarrollo \\
\cline { 2 - 2 } & Requerimientos de la aplicación \\
\hline \multirow{2}{*}{$\begin{array}{l}\text { Producción } \\
\text { Modular }\end{array}$} & $\begin{array}{l}\text { Diseño de la base de datos, del flujo de } \\
\text { datos y de interfaces }\end{array}$ \\
\cline { 2 - 2 } & Definición del plan de pruebas \\
\cline { 2 - 2 } & Creación de aplicación y base de datos \\
\hline Maduración & Ejecutar pruebas modulares \\
\cline { 2 - 2 } Modular & Integrar módulos \\
\cline { 2 - 2 } & Ejecutar pruebas integrales \\
\cline { 2 - 2 } & Corrección y control de mejoras \\
\hline Entrega & $\begin{array}{l}\text { Generación e integración de manuales y } \\
\text { Generamentación }\end{array}$ \\
\cline { 2 - 2 } & $\begin{array}{l}\text { Crear y ejecutar plan de configuración, } \\
\text { despliegue y capacitación de usuarios }\end{array}$ \\
\cline { 2 - 2 } & Liberación \\
\hline
\end{tabular}

Tabla 1 Etapas y artefactos de la metodología de desarrollo

\section{Desarrollo}

Se considera desarrollo al proceso que lleva las cuatro etapas de la metodología, desde la concepción hasta la entrega, así como los entregables para cada etapa.

En la concepción se utilizaron diagramas Business Process Model and Notation (BPMN) con los que se definió el esquema general de los procesos y la conexión entre ellos.

El plan general de desarrollo se plasmó en un diagrama Gantt y para los requerimientos de la aplicación se implementaron diagramas de caso de uso y diagramas de secuencia.

Para la etapa de producción el diseño de la base de datos se realizó en el modelo entidad relación, de forma modular y después se integraron, también se consideró el modelo relacional como lo muestra en la figura 1 con parte del modelo entidad-relación de la base de datos módulo vinculación y la figura 2 con el modelo relacional en el que ya se agrega el módulo de recursos materiales.

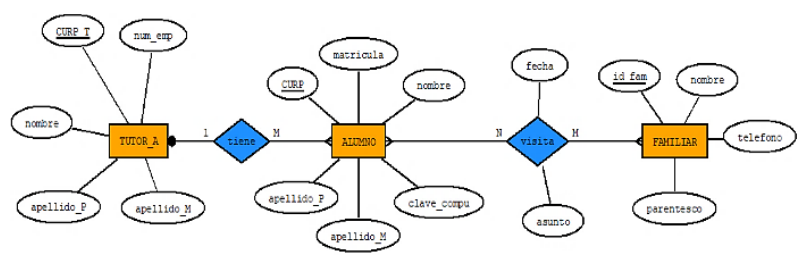

Figura 1 Modelo entidad-relación base de datos módulo vinculación padres de familia

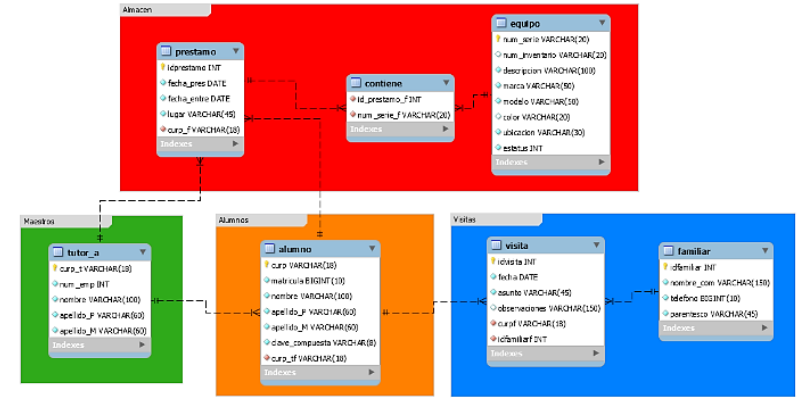

Figura 2 Modelo relacional de base de datos que integra el módulo vinculación padres de familia y el módulo recursos materiales

En esta segunda etapa también se definió el plan de pruebas considerando pruebas modulares e integrales

La creación de la base de datos se dio en mysql, la aplicación se desarrolló utilizando Php, Html5 y Css3. La Figura 3 muestra la interfaz en la que se realiza el alta a un padre de familia que acude a la dirección ISC para tratar algún asunto relacionado con su hijo.

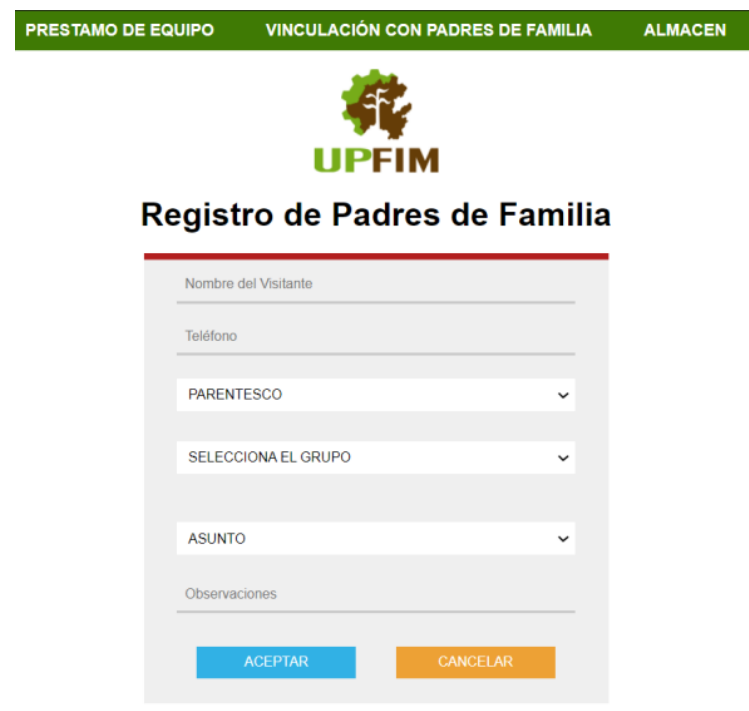

Figura 3 Interfaz del registro de padres de familia en el módulo de vinculación

La Figura 4 contiene la interface en la que se realizan los registros de los equipos que se darán de alta en el módulo recursos materiales
REYNA-ANGELES, Omar, HERNÁNDEZ-TAPIA, Zaila, HERNÁNDEZ-LEÓN, Jonathan Misael y GARCÍA-ROMO, Everardo Said. Desarrollo de aplicación web para automatizar la gestión de procesos del programa educativo de ISC de la UPFIM. Revista de Tecnologías de la Información. 2020 


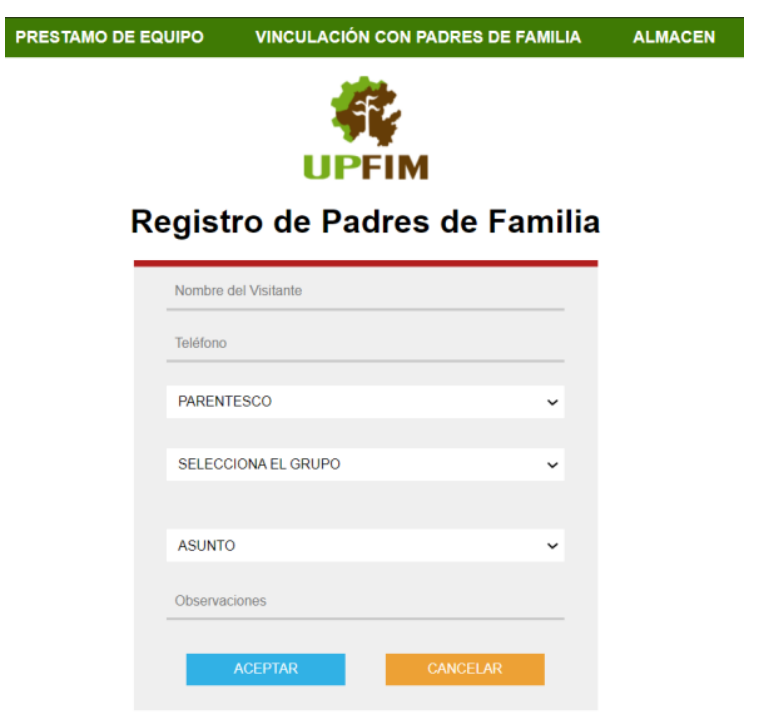

Figura 4 Interfaz del registro de equipos en el módulo recursos materiales

Durante la maduración se realizaron las pruebas modulares establecidas en el plan, se corrigieron las fallas y mejoraron elementos sobre todo en formularios e interfaces, después se integraron los módulos para realizar las pruebas integrales y realizar el mismo proceso de corrección y mejoras que en la fase de pruebas modulares.

Actualmente el desarrollo se encuentra cerrando la fase de maduración, en las mejoras en interfaces y reportes estadísticos, el plan original era que el proyecto se terminara en junio del 2020, sin embargo la contingencia de salud en el país por el covid-19 alteró los planes originales y los esquemas de trabajo, ya que no puede estar desarrollando el equipo en el mismo sitio como se recomienda en la metodología ágil, además la comunicación con el cliente ya no se puede dar de manera regular, se ha adelantado parte de la fase de entrega, se están generando la documentación para usuario, técnica y el plan de despliegue con capacitación. Se realizó un ajuste al plan general de desarrollo se tiene programado concluir en julio y de acuerdo con las condiciones que se presenten, capacitar y liberar en agosto.

\section{Conclusiones}

En este trabajo se diseñó y desarrolló una base de datos, así como un sitio web para automatizar la gestión de los procesos de recursos materiales de la ISC de la UPFIM y la información generada en la vinculación con padres de familia, que permitirá optimizar tiempos y recursos, además de contar con información que permita generar automáticamente estadísticas y reportes para análisis pertinentes y toma de decisiones, una ventaja es que en trabajos futuros se puede dar la integración de otros módulos para gestionar más procesos administrativos que apoyen la formación académica de los alumnos.

\section{Referencias}

Universidad Politécnica de Francisco I. Madero (2016), Programa de Vinculación Escuela Padres de Familia. México.

Remon, T. M. (2014), Desarrollo de Aplicaciones web con php, 1er Ed., México, Editorial Macro.

Remon, T. M. (2016), Diseño Web con Html5 y Css3, 1er Ed., México, Editorial Macro.

Reyna, A. O. (2018). Metodología para Desarrollo de Software Propio de la UPFIM, Ecorfan Journal-Republic of Paraguay, V-4,N-6, 29-35.

Stephen A. White, PHD Derek Miers; BPMN Guía de Referencia y Modelado Edición Digital; 2009; (Disponible en: http://www.futstrat.com/books/BPMN_edicion _espanol.php; Consultado el 16 de febrero del 2020). 